OPEN ACCESS

Edited by:

Emmanuel Stip,

Université de Montréal, Canada

Reviewed by:

Marine Mondino,

INSERM U1028 Centre de Recherche en Neurosciences de Lyon, France

Stephane Potvin,

Université de Montréal, Canada

${ }^{*}$ Correspondence:

Stéphane Raffard

s-raffard@chu-montpellier.fr

Specialty section: This article was submitted to

Schizophrenia,

a section of the journal

Frontiers in Psychiatry

Received: 23 December 2019

Accepted: 13 July 2020

Published: 30 July 2020

Citation:

Raffard S, Lebrun C, Bayard S, Macgregor A and Capdevielle D (2020)

Self-Awareness Deficits of Cognitive Impairment in Individuals With Schizophrenia. Really?

Front. Psychiatry 11:731. doi: 10.3389/fpsyt.2020.00731

\section{Self-Awareness Deficits of Cognitive Impairment in Individuals With Schizophrenia. Really?}

\author{
Stéphane Raffard ${ }^{1,2 *}$, Cindy Lebrun ${ }^{1}$, Sophie Bayard ${ }^{1}$, Alexandra Macgregor ${ }^{2}$ \\ and Delphine Capdevielle $2,3,4$ \\ ${ }^{1}$ Univ Paul Valéry Montpellier 3, Univ Montpellier, EPSYLON EA 4556, Montpellier, France, ${ }^{2}$ Service Universitaire de \\ Psychiatrie Adulte, Hôpital de la Colombière CHU Montpellier, Montpellier, France, ${ }^{3}$ Inserm, U1061, Montpellier, France , \\ ${ }^{4}$ University of Montpellier, Montpellier, France
}

Objective: Misestimation of cognitive functioning has been largely described in individuals with schizophrenia. There is large evidence that correlations between subjectively assessed cognitive functioning and objectively determined cognitive functioning are weak in non clinical individuals and may be more closely related to other psychoaffective or clinical factors than to objective neuropsychological functioning. Surprisingly, no study to date has compared the associations between cognitive complaint and objective measures of cognitive functioning in individuals with schizophrenia and healthy controls. The main objective of this study was to 1) compare cognitive complaint between individuals with schizophrenia and non clinical controls, 2) explore the relationships between cognitive complaint and psychoaffective and clinical factors in the clinical group and 3) compare the relationships between subjective awareness of cognitive functioning and objective neuropsychological assessment in individuals with schizophrenia and non-clinical participants.

Method: In this study 30 individuals with schizophrenia and 20 non-clinical matched controls were included. In addition to objective cognitive measures and subjective cognition assessed by the Subjective Scale To Investigate Cognition In Schizophrenia, measures of psychotic symptoms, depression, and anxiety were included.

Results: Schizophrenia patients reported higher cognitive complaints in comparison with controls. In individuals with schizophrenia, cognitive complaint subscores were differently associated with depression, anxiety, and negative symptoms. When depression was controlled for, the same number of correlations between self-rated measures of cognition and objective measures of cognition were found in both groups, but accuracy of selfassessment of cognition was lower in the schizophrenia group.When the schizophrenia group was divided into a high cognitive complaint group (SZ High CC) and a low cognitive complaint group (SZ Low CC), findings indicated that self-assessment of cognition in the SZ high CC was highly accurate (correlations with large effect sizes). By contrast the SZ low CC group severely misjudge their cognition. 
Conclusion: A significant proportion of patients with schizophrenia can accurately estimate their cognitive skills. Self-awareness of cognitive deficits in individuals with schizophrenia is an heterogenous phenomenon and misestimation of cognitive functioning might have been overestimated, partly due to secondary psychoaffective factors. Caution is warranted before jumping to the conclusion that all individuals with schizophrenia misjudge their cognitive functioning.

Keywords: schizophrenia, cognitive complaint, self-awareness, cognitive functioning, heterogeneity

\section{INTRODUCTION}

Metacognition can be considered as an umbrella term describing a broad set of processes relating to the development of selfawareness (1) and ability to self-assess and self-reflect upon one's emotional and cognitive experiences and abilities [See (2) for a review and discussion of the term applied to the field of clinical psychology]. Metacognitive deficits are considered by many authors as a core deficit of the schizophrenia spectrum disorders $(3,4)$. Metacognition impairments have been explored in individuals with schizophrenia in a large set of processes including reasoning and memory biases (5), beliefs (6), autobiographical memory (7), or insight into illness (8), to name but a few. This latter metacognitive ability, also named clinical insight (8), has been particularly studied in schizophrenia mainly because poor awareness into illness is highly prevalent in this population (9), leading to poor prognosis, and in association with self-stigma to higher risk of suicide (10) and depression (11). In this study we will focus on metacognitive awareness of cognitive functioning corresponding to the adequacy between the subjective report of cognitive functioning and objective cognitive performances.

In comparison with clinical insight, relatively few studies have examined these associations in individuals with schizophrenia despite evidence that cognitive impairments in the disease are present in most of cognitive domains including working and episodic memories, attention processes, abstraction, and executive functioning $(12,13)$. Several measures have been previously used to measure self-report of cognitive functioning in individuals with schizophrenia. Among them the Cognitive Failures Questionnaire [CFQ; (14)], the Measure of insight into cognition-self-rated [MIC-SR; (15)], or the subjective scale to investigate cognition in schizophrenia [SSTICS, (16)] which is the most commonly used tool to assess self-awareness of cognitive functioning in this mental disorder $(17,18)$.

To date, only one meta-analysis has been published on this subject (18). Several findings emerged from this meta-analysis and can be summarized as follows: 1) individuals with schizophrenia reported higher cognitive complaints than nonclinical controls; 2) modest associations between measures of objective and subjective cognition were found (the higher for problem-solving domain) 3) these relationships were higher when the study used the SSTICS to measure self-reported cognitive functioning in comparison with other scales, and 4) a weak association was found between depressive symptoms and cognitive complaint. Interestingly, several studies have been published after this meta-analysis, reporting as a whole similar results (19-22). However, and surprisingly, none of these studies about metacognitive awareness of cognitive functioning in schizophrenia examined and compared correlations between measures of objective and subjective cognition in a comparison group of non-clinical controls. For all that, previous studies in non-clinical individuals have constantly reported weak correlations between subjectively assessed cognitive functioning and objectively determined cognitive functioning ( $r$ generally ranging from 0.20 to 0.30 ) (23-25). In addition, it has been suggested that subjective cognitive complaint are more likely related to psychoaffective variables such as depression and anxiety rather than to psychotic symptoms or concurrent cognitive impairments in schizophrenia $(21,26)$. Note that this association was also previously reported in aging (23) or neurological disorders (27).

Consequently, the main objective of this study is to 1) compare cognitive complaint between individuals with schizophrenia and non clinical controls, 2) explore the relationships between cognitive complaint and psychoaffective and clinical factors in the clinical group, and 3) compare the relationships between subjective awareness of cognitive functioning and objective neuropsychological assessment in individuals with schizophrenia and non-clinical participants.

\section{MATERIALS AND METHODS}

\section{Method}

\section{Participants}

Thirty outpatients with schizophrenia and 20 healthy controls completed this study. Inclusion criteria for patients were: (a) age between 18 and 65 years, (b) a DSM-5 diagnosis of schizophrenia, (c) adequate proficiency in French. Exclusion criteria for all participants were: (a) known neurological disease, (b) history of learning disability/developmental disorder, or (c) substance abuse in the past month (other than cannabis or tobacco). Controls were recruited from the general population and had the additional exclusion criterion that they had no personal lifetime history of any psychosis or affective disorders diagnosis. Controls with a family member with bipolar or schizophrenia disorders were also excluded. The comparison control group was selected to match the schizophrenia group on sex, age, and education-level variables. The demographic data are shown in Table 1. 
TABLE 1 | Demographics and clinical characteristics of schizophrenia patients and controls.

\begin{tabular}{|c|c|c|c|c|}
\hline & Schizophrenia $(\mathbf{N}=30)$ & Controls $(N=20)$ & Statistics & $P$ value \\
\hline \multicolumn{5}{|l|}{ Demographic data } \\
\hline Sex (female) & 11 & 7 & $\chi^{2}=0.90$ & 0.57 \\
\hline Age (years) & $33.73 \pm 9.48[21-56]$ & $32.95 \pm 13.34[18-53]$ & $t=0.24$ & 0.80 \\
\hline Education Level (years) & $11.46 \pm 3.39[6-22]$ & $12.3 \pm 2.20[9-16]$ & $t=-0,96$ & 0.33 \\
\hline \multicolumn{5}{|l|}{ Clinical variables } \\
\hline Duration of illness & $9.15 \pm 9.46[2-34]$ & - & - & - \\
\hline PANSS & $72.40 \pm 12.11[38-88]$ & - & - & - \\
\hline \multicolumn{5}{|l|}{ Total score } \\
\hline Positive symptoms & $16.63 \pm 5.48[7-26]$ & - & - & - \\
\hline Negative symptoms & $21.23 \pm 5.89[12-35]$ & - & - & - \\
\hline General psychopathology & $34.16 \pm 6.45[18-47]$ & - & - & - \\
\hline $\mathrm{BDI}$ & $16.30 \pm 9.18[0-32]$ & $8.20 \pm 4.16[2-17]$ & $t=2.72$ & 0.009 \\
\hline STAI-S & $37.06 \pm 11.36[20-69]$ & $33.30 \pm 7.82[23-47]$ & $t=1.06$ & 0.29 \\
\hline STAI-T & $41.10 \pm 11.64[20-72]$ & $40.45 \pm 9.44[24-59]$ & $t=0.20$ & 0.83 \\
\hline \multicolumn{5}{|l|}{ SSTICS } \\
\hline Total score & $25.56 \pm 9.10[1-46]$ & $22.55 \pm 7.87[7-40]$ & $F=5.12$ & 0.02 \\
\hline Working memory & $3.03 \pm 1.86[0-7]$ & $3.35 \pm 1.87[1-8]$ & $F=0.62$ & 0.54 \\
\hline Explicit memory subscale & $11.70 \pm 3.38[0-21]$ & $9.35 \pm 3.93[3-15]$ & $F=6.14$ & 0.04 \\
\hline Attention subscale & $6.46 \pm 4.23[0-17]$ & $6.20 \pm 2.83[1-12]$ & $F=2.05$ & 0.13 \\
\hline Executive functions subscale & $2.83 \pm 2.32[0-9]$ & $2.20 \pm 1.90[0-7]$ & $F=1.63$ & 0.20 \\
\hline
\end{tabular}

N, sample size; Mean \pm standard deviation [range]; BDI-II, Beck Depression Inventory; STAI-T, trait State-Trait Anxiety Inventory; STAI-S, state State-Trait Anxiety Inventory; PANSS, Positive and Negative Syndromes Scale total score; Bold font indicates a significant correlation $(p<0.05)$.

\section{Measures}

\section{Clinical Variables}

In the schizophrenia sample, severity of symptoms was evaluated with the positive and negative syndrome scale (PANSS) for schizophrenia (28). For all participants current emotional status was assessed using the Beck Depression Inventory-II (BDI-II) (29) and the Spielberger State Trait Anxiety Inventory (STAI Trait and STAI State) (30).

\section{Cognitive Complaint}

\section{Subjective Scale to Investigate Cognition in Schizophrenia (SSTICS)}

Cognitive complaint was measured by the SSTICS (16). Twentyone items focus on four cognitive domains: memory, attention, executive functions, and praxia. Each item is rated on a five-point Likert scale ranging from "never" to "very often" and refers to how often a problem occurs. A higher score suggests higher cognitive complaint. The scale has good internal consistency $(\alpha=$ $0.86)$ and test-retest reliability $(r=0.80 ; 16)$. For this study, we focused on working memory, explicit memory, attention, and executive functions subscales.

\section{Objective Cognitive Measures}

We used a computer-based neuropsychological battery, Zimmermann and Fimm (31)'s attention test battery (Testbatterie zur Aufmerksamkeitsprüfung-TAP) to evaluate executive and attentional processes. This well-validated test battery provides normative data for adults. The task consists of simple, easily distinguishable stimuli to which the patient's motor response is recorded. A button box with millisecond accuracy was used to capture reaction times (RTs) and record responses (false alarms and/or omissions). Several subscales of the TAP were used and administered in random order: the 2Back Task (Updating), the Go/No-Go Task (Inhibition), the
Flexibility Task (Shifting), the Divided Attention Task (Dual task coordination), and the Vigilance Task (Vigilance) to assess executive and attentional processes. To assess verbal learning we used the Grober and Buschke verbal learning test (32).

\section{Executive and Attentional Processes Go/No-Go Task}

This subtest measures the ability to suppress a response in the presence of irrelevant stimuli as well as the response latency during stimulus selection. In this study, a version of the Go-nogo task with a higher memory load with five stimuli, squares with different textures, was realized. One $3 \times 3 \mathrm{~cm}$ square appears in the middle of the screen. There are two target and three nontarget stimuli. The subject has to press the button on the presentation of a target and not to press on the presentation of a nontarget. A total of 60 trials were presented. The median RT served as primary measure.

\section{Flexibility Task}

In this subtest the flexibility of focused attention is tested by a mental alternation between two sets of targets. There are two alternatives for testing, a verbal and a non-verbal version. In this study, we used the verbal version in which the sets of target consist of letters and numbers. For testing, two stimuli, one from each set, are presented simultaneously and randomly on the left or the right side of the fixation point. From one presentation to the other, the target changes from letter to number and vice versa. The subject has to press as quickly as possible the key on the side of the target (left or right). The dependent measure was the median RT. The dependent measure was the median RT.

\section{The 2-Back Task}

The 2-Back is a verbal working memory task in which series of consonants are presented visually, one every 3,000 ms (2,500 ms stimulus and $500 \mathrm{~ms}$ interstimulus interval). Participants were 
asked to make a yes/no response following each consonant, determining whether it was the same as or different from the consonant presented two earlier (e.g., f, N, b, N, B, K, b, k, N, G...). Responses were made using two keys on the keyboard of a laptop computer. Capitalization was randomized and each consonant block contained 33\% targets. The effects of the 2Back condition were obtained by comparing performance to the 0 -Back, a control condition. The 0 -Back task included nine consonants presented at the same rate, with $33 \%$ targets. Participants responded yes when a predetermined target consonant ("X" or " $\mathrm{x}$ ") appeared and no for other consonants. In total, there were four 6-min series of four 0 -Back/2-Back cycles each. As the dependent variable, we computed the difference in RT between the 0-back and 2-back condition, which should reflect the specific working memory-related task demands (storage and updating).

\section{Divided Attention Task}

The divided attention task required the simultaneous performance of spatial and auditory tasks. The spatial task comprised the identification of a pre-determined visual configuration assembled from crosses. For the auditory task, the participants were instructed to detect a "beep/bop" alternation in a repetitive sequence. Number of omission errors and commission errors were recorded for further analysis.

\section{Vigilance Task}

This task assesses the subject's ability to sustain attention for long periods of time. Vigilance was tested over a period of $30 \mathrm{~min}$ at a rate of one critical stimulus per minute This subtest presents the subject with a series of high and low tones $(440$ and $1,000 \mathrm{~Hz})$ in regular alternation ("di da di da..."). The subject must discover the appearance of an irregularity in the sequence. The target stimuli have a low frequency of appearance. The median RT was the dependent variable.

\section{Alertness Task}

This task concerns simple RT measurements, in which a cross appears on the monitor at randomly varying intervals and to which the subject should respond as quickly as possible by pressing a key. The task also concerns the RT following the presentation of an audible warning signal, which allows the calculation of a phasic alertness index (improvement of the reaction speed following the presentation of the warning signal). The phasic alertness index was the dependent variable.

\section{The Grober and Buschke Verbal Learning Test}

The test is composed of 16 words belonging to 16 different semantic categories and presented on cards containing four words at a time (32). The patient is asked to read aloud and to point to each item (e.g. herring) following the examiner's indication of a category (e.g., "what is the name of the fish?"). When the four items are thus learned, the card is removed and the patient is given the four categories as a cued immediate recall. If the patient is enable to respond, the card is shown again and the same steps as above are carried out until every item is encoded. The learning phase is achieved when all or nearly all the items have been encoded. The test includes therefore successively an encoding control phase, an immediate cued recall, then three successive free and cued recall trials, a delayed free and cued recall phase $(20 \mathrm{~min})$, followed by a recognition phase. For both free recall 3 and delayed free recall, the amount of correctly recalled items was registered.

\section{Procedure}

Participants' recruitment took place within the University Department of Adult Psychiatry in Montpellier CHU. All participants were tested individually in two sessions: the first session included a clinical assessment (PANSS, SSTICS, BDI-2, STAI) conducted by a fully trained clinical psychologist; 1 or 2 days following the first session, the second session was completed involving the administration of the neuropsychological tests by a fully trained clinical neuropsychologist. The clinical neuropsychologist was blind of the SSTICS scores of the participants. The study was approved by the relevant ethical review board [Comité de Protection des Personnes (CPP) Ile de France IX] on January 18, 2010. Written informed consent was obtained from all participants.

\section{Statistical Analyses}

All statistical analyses were performed with IBM SPSS software version 24.0. The continuous variables were expressed as mean \pm standard deviation and categorical variables as frequency and percentage. Prior to analysis, all data were examined for normality using the Shapiro-Wilk test. The Shapiro-Wilk tests revealed non-normal distributions for executive and attentional processes $(p<0.05)$. Therefore, the Kruskal-Wallis test was chosen as a non-parametric alternative to the analysis of variance tests. The median RT for all executive and attentional processes, the number of omission errors and commission errors for divided attention and the phasic alertness index were examined using $H$ tests. Post hoc analyses were performed using the MannWhitney $\mathrm{U}$ test to determine the directions of the significant $H$ test results. The significance level was set at $\mathrm{p}<0.05$. Furthermore, effect sizes (Cohen's r) of all significant group differences were computed. Cohen's $r$ was chosen as it does not rely on the normality assumption. Based on Cohen's criteria for $r=0.10$ indicates a small effect, 0.30 indicates a medium effect, and 0.50 indicates a large effect (33).

All other variables exhibited normal distributions ( $p>0.05$ ). Therefore, we conducted parametric analyses of variance (ANOVAs) and Student's t-tests to assess group differences for continuous variables. The Chi-square test was performed for categorical variables. In addition, we calculated the eta squared $\eta^{2}$ and the Cohen's $d$ ' as measure of the effect size. The effect size was considered small $\left(\eta^{2}=0.01 ; d=0.2\right)$, medium $\left(\eta^{2}=0.06 ; d=\right.$ $0.5)$, or large $\left(\eta^{2}=0.14 ; d=0.8\right)$ according to Cohen (33).

To test for potential relationships between variables in groups, we calculated partial correlations (with rs of.10,.30, and.50 defined as small, medium, and large effect size, respectively, 33). In addition, bivariate correlations between cognitive complaint and clinical characteristics for schizophrenia patients were calculated according to Pearson's p. The $\alpha$-level was set to $\mathrm{p}=0.05$. 
Finally, additional exploratory statistical analyses were performed in the clinical group of individuals with schizophrenia in order to compare objective measures of cognitive functioning regarding their level of cognitive complaint. For this, the schizophrenia group was divided into a high cognitive complaint group and a low cognitive complaint group, based on a median split of the SSTICS total score. We also explored the relationships between objective and subjective measures of cognitive functioning in these both subgroups of patients.

\section{RESULTS}

\section{Demographics}

Groups did not differ significantly in terms of age $\left(\mathrm{t}_{(48)}=0.24\right.$, $\mathrm{p}=0.80)$, sex $(\chi 2=0.90, \mathrm{p}=0.57)$, education level $\left(\mathrm{t}_{(48)}=-0,96\right.$, $\mathrm{p}=0.33$ ), or state and trait anxiety (respectively, $\mathrm{t}_{(48)}=1.06, \mathrm{p}=$ $0.29, \mathrm{t}(48)=0.20, \mathrm{p}=0.83$ ). Level of depressive symptoms was significantly higher in schizophrenia patients than in control participants $\left(\mathrm{t}_{(48)}=2.72, \mathrm{p}=0.009, d^{\prime}=0.86\right)$. Table 1 presents demographic and clinical characteristics of the participants.

\section{Do Patients With Schizophrenia Differ From Control Participants on Cognitive Complaint?}

Schizophrenia Patients Versus Control Group Comparisons Given that patients and controls significantly differ on depressive symptoms, the BDI-II was considered as a covariate in the group comparison analysis. A multivariate ANCOVA was used to examine group differences in cognitive complaint (Table 1). The results showed significant difference on the SSTICS total $(\mathrm{F}=3.99, \mathrm{p}=0.02, \eta 2=0.14)$ and explicit memory scores $(\mathrm{F}=$ $3.14, \mathrm{p}=0.04, \eta 2=0.11)$ between schizophrenia patients and control participants, indicating that patients have a higher cognitive complaint. No significant difference was noticed for the other cognitive domains (working memory, attention, and executive functions) rated on the SSTICS (all ps > 0.05).

\section{Relationships of SSTICS Scores to Clinical Measures for Schizophrenia Participants}

Table 2 shows correlations between each domains of cognitive complaint and other clinical measures in patients.

\section{Overall Cognitive Complaint}

Higher overall cognitive complaint in schizophrenia patients is associated with longer disease duration, and higher levels of both depressive symptoms and trait anxiety. Higher overall cognitive complaint in schizophrenia patients is associated with lower levels of negative symptoms. The size of this association was moderate to large.

\section{Complaint of Memory Problems}

Higher complaint of working memory problems in schizophrenia patients is associated with higher levels of both state and trait anxiety. Higher complaint of explicit memory problems in schizophrenia patients is associated with longer disease duration and higher levels of both depressive symptoms and trait anxiety. The size of this association was moderate.

\section{Complaint of Attention Problems}

Higher complaint of attention problems in schizophrenia patients is associated with longer disease duration and higher levels of depressive symptoms and both state and trait anxiety. Higher complaint of attention problems in schizophrenia patients is associated with lower levels of negative symptoms. The size of this association was moderate to large.

\section{Complaint of Executive Problems}

Higher complaint of executive problems in schizophrenia patients is only associated with higher levels of trait anxiety. The size of this association was moderate.

\section{Relationships of SSTICS Scores to Objective Cognitive Measures for Schizophrenia Patients and Controls}

As mentioned above, groups differ according severity of depressive symptoms rated on the BDI. The following correlational analyses were therefore controlled for depressive symptoms.

\section{Schizophrenia Patients}

Higher overall cognitive complaint in schizophrenia patients was significantly associated with lower phasic attention $(r=.39$, $\mathrm{p}=0.034)$

Higher complaint of explicit memory problems in schizophrenia patients was significantly associated with lower phasic attention $(\mathrm{r}=0.46, \mathrm{p}=0.013)$ and lower vigilance performances $(\mathrm{r}=-.34, \mathrm{p}=0.029)$.

TABLE 2 | Pearson correlations between cognitive complaint domain and clinical measures for schizophrenia patients.

\begin{tabular}{|c|c|c|c|c|c|}
\hline Clinical variables & sSTICS-total & SSTICS-working memory & SSTICS-explicit memory & SSTICS-attention & SSTICS-executive functions \\
\hline Disease duration & $0.56^{\star *}$ & 0.22 & $0.45^{\star \star}$ & $0.48^{\star *}$ & 0.23 \\
\hline BDI-II & $0.40^{*}$ & 0.13 & $0.48^{\star *}$ & $0.36^{\star \star}$ & 0.26 \\
\hline STAI-S & 0.27 & $0.42^{\star *}$ & 0.05 & $0.48^{\star \star}$ & 0.16 \\
\hline STAI-T & $0.62^{\star *}$ & $0.49^{\star *}$ & $0.33^{*}$ & $0.65^{\star \star}$ & $0.46^{\star *}$ \\
\hline PANSS & -0.23 & -0.30 & -0.06 & -0.25 & -0.31 \\
\hline \multicolumn{6}{|l|}{ Total score } \\
\hline Positive symptoms & -0.06 & -0.08 & -0.08 & -0.06 & -0.33 \\
\hline Negative symptoms & $-0.40^{\star}$ & -0.33 & -0.27 & $-0.37^{\star}$ & -0.30 \\
\hline General psychopathology & -0.08 & -0.28 & -0.09 & -0.21 & -0.06 \\
\hline
\end{tabular}

SSTICS, Subjective Scale to Investigate Cognition in Schizophrenia; PANSS, Positive and Negative Syndromes Scale total Score; BDI-II, Beck Depression Inventory, STAI-T, trait StateTrait Anxiety Inventory; STAI-S, state State-Trait Anxiety Inventory; ${ }^{*} p<.05$; ${ }^{* *} p<.01$; (medium and large effect are shown in bold print) 
Higher complaint of working memory problems in schizophrenia patients was significantly associated with lower phasic attention $(\mathrm{r}=$ $.34, \mathrm{p}=0.032)$ and higher updating deficits $(\mathrm{r}=.45, \mathrm{p}=0.017)$. Overall, the size of these associations was moderate.

\section{Controls}

Higher overall cognitive complaint in controls was significantly associated with lower inhibition performances $(r=-0.49$, $\mathrm{p}=0.014)$.

Higher complaint of explicit memory problems in controls was significantly associated with lower memory performances $(\mathrm{r}=-0.43, \mathrm{p}=0.030)$.

Higher complaint of working memory problems in controls was significantly associated with lower higher updating deficits $(\mathrm{r}=.44, \mathrm{p}=0.026)$

Higher complaint of executive problems in controls was significantly associated with lower inhibition performances $(r=$ $-0.46, \mathrm{p}=0.020)$.

Higher complaint of attention problems in controls was significantly associated with lower inhibition performances $(\mathrm{r}=$ $-0.41, \mathrm{p}=0.034)$. Overall, the size of these associations was moderate.

\section{Additional Exploratory Analyses \\ Schizophrenia Patients With High and Low Cognitive Complaint versus Control Group Comparisons}

The schizophrenia group was divided into a high cognitive complaint (SZ High CC) group and a low cognitive complaint group (SZ Low CC), based on a median split of the SSTICS total score. Further analysis of cognitive complaint domains was thus performed on 14 SZ High CC, 16 SZ Low CC, and 20 controls. These groups did not differ in terms of age $(F=2,46, p=0.09)$, sex $(\chi 2=2.04, \mathrm{p}=0.36)$, education level $(\mathrm{F}=0.47, \mathrm{p}=0.62)$, or both state $(\mathrm{F}=1.05, \mathrm{p}=0.35)$ and trait anxiety scores $(\mathrm{F}=2,27 \mathrm{p}=$ $0.11)$. However, the groups differed on BDI-II score $(\mathrm{F}=4.15 \mathrm{p}=$ 0.02). SZ High CC $(M=19.78 \pm 7.66)$ had significantly higher depressive symptoms compared to both controls $(\mathrm{M}=9.20 \pm 5.13$, $\mathrm{p}<.001)$ and SZ Low CC groups $(\mathrm{M}=13.25 \pm 9.53, \mathrm{p}=0.04)$.
However, SZ Low CC and controls did not differ. The comparison analyses were therefore controlled for depressive symptoms.

Table 3 displays clinical data for SZ High CC and SZ Low CC groups. SZ High CC and SZ Low CC groups differed in terms of age $(\mathrm{t}=-2,77, \mathrm{p}=0.01)$, disease's duration $(\mathrm{t}=-4,39, \mathrm{p}<0.001)$, and severity of depressive symptoms $(\mathrm{t}=-2.04, \mathrm{p}=0.04)$.

\section{Do Schizophrenia Patients High and Low Cognitive Complaint Groups Differ Across Objective Cognitive Measures?}

Kruskal-Wallis tests revealed significant differences between patients with SZ and control participants on objective cognitive measures. The Post hoc comparisons (Mann-Whitney U tests) of the SZ groups to controls and corresponding effect sizes indicated impairments on individual test measures ranging from medium to large effect sizes. In comparison to the control participants, the SZ Low CC and SZ High CC groups showed significantly poorer performances with regard to inhibition processes (large effects for Go/no-go RT), flexibility, (large effects for flexibility RT), storage and updating (medium to large effects for n-back RT), as well as for attentional processes (medium effects for vigilance RT and large effects for the divided attention omissions errors), and memory (large effects for free recall 3 and delayed free recall). The groups did not differ on phasic alertness. Considering subgroups of patients' analyses, the SZ Low CC and SZ High CC groups did not differ significantly from each other on any of the neuropsychological measures (Table 4).

\section{Relationships of SSTICS Scores to Objective Cognitive Measures for Schizophrenia Patients High and Low Cognitive Complaint Groups}

As described above in Table 3, SZ High CC and SZ Low CC groups differed in terms of age, disease's duration, and severity of depressive symptoms. The following correlational analyses were therefore controlled for age, disease's duration, and depressive symptoms.

TABLE 3 | Demographics and clinical characteristics of schizophrenia patients regarding their level of cognitive complaint.

\begin{tabular}{|c|c|c|c|c|}
\hline & High cognitive complaint * $(N=14)$ & Low cognitive complaint * $(N=16)$ & Statistics & $P$ value \\
\hline \multicolumn{5}{|l|}{ Demographic data } \\
\hline Sex (female) & 7 & 4 & $\chi 2=2.04$ & 0.36 \\
\hline Age (years) & $36.35 \pm 10.30[27-56]$ & $29.68 \pm 6.67[21-46]$ & $t=-2,77$ & 0.01 \\
\hline Education Level (years) & $11.35 \pm 3.02[6-17]$ & $11.56 \pm 3.77[7-22]$ & $t=0.16$ & 0.87 \\
\hline \multicolumn{5}{|l|}{ Clinical variables } \\
\hline Duration of illness & $15.05 \pm 10.37[3-34]$ & $3.59 \pm 3.03[2-9]$ & $t=-4,39$ & $<0.001$ \\
\hline $\mathrm{BDI}$ & $19.78 \pm 7.66[0-37]$ & $13.25 \pm 9.53[0-26]$ & $t=-2.04$ & 0.04 \\
\hline STAI-S & $38.42 \pm 11.95$ [20-69] & $35.87 \pm 11.07[20-54]$ & $t=-0.60$ & 0.54 \\
\hline STAI-T & $45.42 \pm 10.52[20-72]$ & $37.31 \pm 11.54[20-52]$ & $t=-2.00$ & 0.05 \\
\hline PANSS & $71.28 \pm 10.31[54-87]$ & $73.37 \pm 13.75[38-88]$ & $t=-0.46$ & 0.64 \\
\hline \multicolumn{5}{|l|}{ Total score } \\
\hline Positive symptoms & $16.55 \pm 5.37[9-26]$ & $16.68 \pm 5.74[7-26]$ & $t=-0.05$ & 0.95 \\
\hline Negative symptoms & $20.14 \pm 4.68[13-28]$ & $22.18 \pm 6.79[12-35]$ & $t=-94$ & 0.35 \\
\hline General psychopathology & $33.78 \pm 6.60[20-47]$ & $34.50 \pm 6.53[18-44]$ & $t=-0.29$ & 0.76 \\
\hline
\end{tabular}

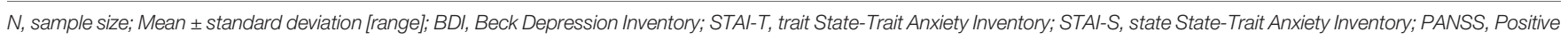
and Negative Syndromes Scale total score; Bold font indicates a significant correlation $(p<0.05)$

*The schizophrenia group was divided into a high cognitive complaint (SZ High CC) group and a low cognitive complaint group (SZ Low CC), based on a median split of the SSTICS total score. 


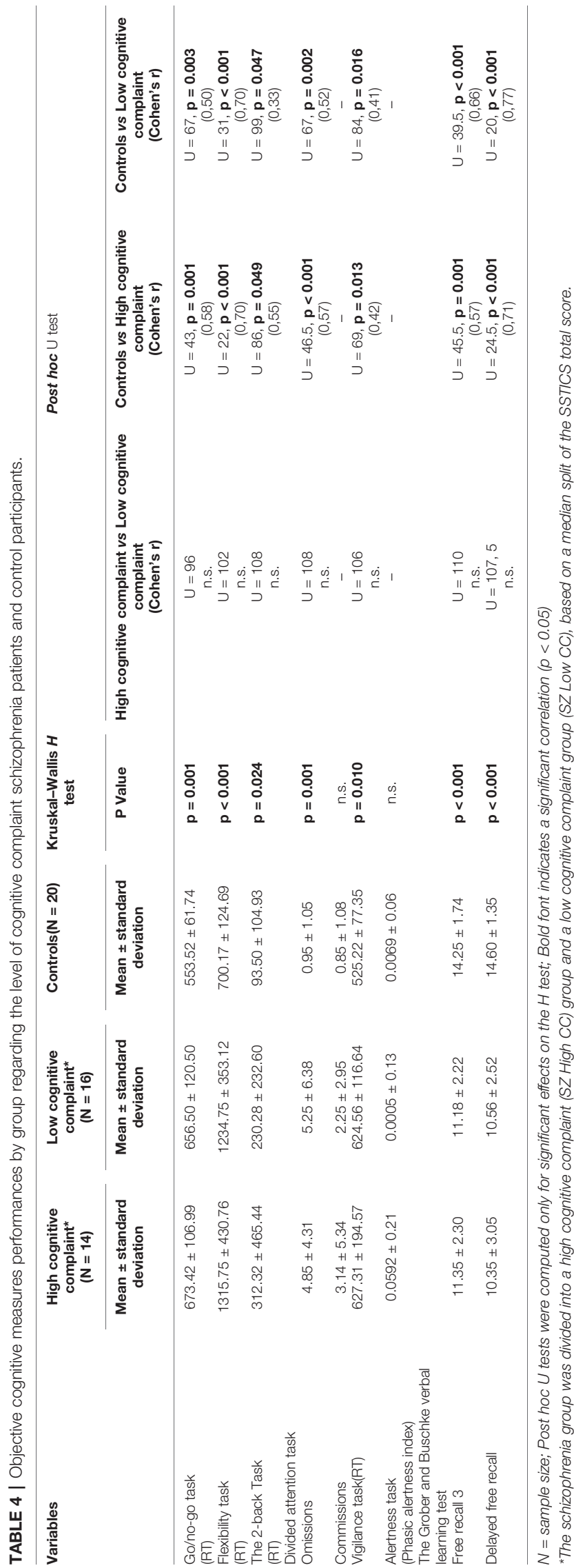

\section{Schizophrenia High Cognitive Complaint}

Higher overall cognitive complaint in SZ High SA was significantly associated with lower updating deficits $(r=.66$, $\mathrm{p}=0.028$.

Higher complaint of explicit memory problems in SZ High SA was significantly associated with lower memory abilities $(\mathrm{r}=$ $-0.67, \mathrm{p}=0.024)$.

Higher complaint of working memory problems in SZ High SA was significantly associated with higher inhibition deficits $(\mathrm{r}=-0.63, \mathrm{p}=0.037)$. Overall, these relationships have large effect sizes.

\section{Schizophrenia Low Cognitive Complaint}

There were no significant correlations between SSTICS scores and objective cognitive measures.

\section{DISCUSSION}

The aim of our study was to 1) compare cognitive complaint between individuals with schizophrenia and non clinical controls, 2) explore the relationships between cognitive complaint and psychoaffective and clinical factors in the clinical group, and 3) compare the relationships between subjective awareness of cognitive functioning and objective neuropsychological assessment in individuals with schizophrenia and non-clinical participants

First, our findings indicated that at group level individuals with schizophrenia reported higher subjective cognitive difficulties when depression was controlled for regarding some (SSTICS total score and the explicit memory subscore) but not all SSTICS subscores. Indeed, no differences between groups were found for working memory, attention, and executive functions subscores of the SSTICS. Concerning increased subjective cognitive complaint in patients in comparison with controls, this result is in accordance with the findings of the meta-analysis of Potvin et al. (18) who have observed a similar results for the SSTICS total score.

Regarding the second aim of our study, significant relationships were found between psychoaffective measures (state anxiety and depression), duration of illness, psychotic symptoms (i.e. negative symptoms), and cognitive complaint in the clinical group. Regarding psychoaffective factors this is in accordance with some $(20,21,26,34)$ but not all studies on this subject $(35,36)$ in schizophrenia. In our study, and similarly to the study of Bayard et al. (26) we used the Beck depression Inventory 2 which is a self-rated measure of depression whereas Potvin et al. (36) for example used the Calgary Depression Scale for Schizophrenia which consist on a semi-structured interview (37). Similarly, the STAI is also a self-reported measure of anxiety. Taken together, this can explain our findings and confirm the view that subjective cognitive complaints are related to depression and psychoaffective variables in individuals with schizophrenia as it has been consistently found in other populations such as chronic pain (38), older adults (39 for a review), neurological disorders such as multiple sclerosis (40) or mild cognitive impairment (41) particularly when self-reported measures of depression or anxiety are used. 
For the third aim of our study and as the two groups (controls $v s$. individuals with schizophrenia) differed according to the severity of depressive symptoms, we computed partial correlations between the subtests of the SSTICS and the corresponding objective neuropsychological measures controlling for depression. For the overall sample of schizophrenia patients, five significant correlations with a moderate effect size were found. Similarly regarding the control group, five significant correlations were found, also with a moderate effect size. However, whereas significant correlations were related to three cognitive domain in patients (overall cognitive functioning, working memory, and explicit memory), five scores of the SSTICS were associated with objective cognitive measures in controls suggesting better and more accurate self-awareness of cognitive functioning in healthy controls in comparison with individuals with a diagnosis of schizophrenia.

However, we decided to further explore the relationships between subjective measure of cognition and objective cognition in the schizophrenia group. The schizophrenia group was divided into a high cognitive complaint (SZ High CC) group and a low cognitive complaint group (SZ Low CC), based on a median split of the SSTICS total score. If the two groups did not differ significantly from each other on any of the neuropsychological measures, they differed in terms of age, disease's duration, and severity of depressive symptoms, the SZ High CC group having higher depressive symptoms and a longer duration of illness than the SZ low CC. We found in the SZ High CC group three correlations between the SSTICS scores and objective cognitive functioning with large effect sizes. It is important to note that even if five correlations were found in the control group, effect sizes were only moderate. Additionally, as in the control group the SSTICS scores matched adequately with the objective neuropsychological measures (i.e. explicit memory subscore of the SSTICS with the Z-score of the Grober and Buscke verbal learning test and inhibition with working memory subscore of the SSTICS). It is important to note that unlike to past previous studies, neuropsychological tools were chosen in our study to fit adequately and specifically with the different SSTICS subsets in order that each test can correspond with a domain evaluated by the SSTICS. In our study we used a relatively large number of specific cognitive processes compared to other past studies on this topic which often used measures of global functioning. It is possible that the convergent validity of our cognitive assessment with the SSTICS might have increased the validity of participant's estimation of their own cognitive functioning with actual performance ranking.

Finally, no correlations between SSTICS scores and any of the neuropsychological scores were found in the low SZ CC group. This suggests that if some patients exhibit a good self-awareness of cognitive functioning, a significant proportion have clear neurocognitive insight deficits. Nevertheless and from a clinical perspective, several studies indicated that impaired neurocognitive insight is not a barrier to participate to a cognitive remediation program. For example Burton \&
Twamley (19) showed that among individuals with impaired neurocognitive insight, those who participated to a cognitive remediation program performed better than those who did not receive treatment. Furthermore, rather than focusing on cognitive complaint (strongly associated with depression and emotional distress), focusing on functional disabilities could be a better pathway to promote a personalized approach leading to sustainable functional changes (42), particularly in individuals with impaired abilities to recognize and manage one's own cognitive functioning.

Taken together, our results suggest that even if we must acknowledge that the schizophrenia group as a whole exhibits relatively poorer self-awareness of cognitive functioning in comparison to the non-clinical group, one cannot conclude that they have strong self-awareness deficits. Indeed, a similar number of correlations between subjective scores of the SSTICS and objectives measures of cognition with moderate to large effect sizes were found in the two groups. In this context, the state that individuals with schizophrenia « did a very poor job of accurately classifying their cognitive status " compared to healthy controls (43) is inaccurate and an oversimplification of the neurocognitive insight phenomenon. Indeed, our findings clearly indicate that schizophrenia patients should not be considered as an homogeneous group as it is too often the case. When we split our clinical group in a high and a low cognitive complaint groups, individuals in the SZ High CC group had accurate and high selfawareness of their cognitive status, and even larger to what it is usually reported in healthy controls (44). As a consequence, if there is good evidence for significant cognitive heterogeneity in schizophrenia (45), it appears that subjective cognition in schizophrenia is also clearly heterogeneous and the extent of heterogeneity is yet to be investigated in larger samples.

From a theoretical perspective, and even if metacognition deficits commonly occur in schizophrenia (2), our findings support the view that some individuals with schizophrenia have preserved metacognitive abilities. As pointed out by some authors $(3,4)$, an individual's capacity for metacognition cannot be assess categorically or dichotomize as present or absent. Instead, similarly to nonclinical individuals, metacognition is a multidimensional construct varying in individuals with schizophrenia along several and independent measurable dimensions.

Our study has some limitations. One of the main limitations in the present study is the small sample size of our two groups of participants. In addition, we decided to divide the schizophrenia group $(\mathrm{n}=30)$ into those with high and low cognitive complaints. Even if we controlled for potential confounds, we cannot exclude that we have produced false-positive results, and/or over-estimated the magnitude of the associations found in our study. Thirdly, the use of a median split approach for subdividing patients into subgroups of high and low cognitive complaint is not optimal. Future studies should perform correlational analyses between objective and subjective cognitive functioning in subgroups of individuals with schizophrenia patients regarding their cognitive complaint in a larger sample and based on well-defined cut-off values on the SSTICS. Fourth, anxiety was assessed with the STAI in 
our two groups, which is not optimal to measure anxiety, even more so in individuals with schizophrenia. Further studies involving wellvalidated scales such as the Scale of Anxiety Evaluation are needed (46). Finally, there is now evidence that outside psychoaffective states performance on measures of neurocognition in schizophrenia are to a considerable extent due to secondary factors such as poor motivation, fatigue, and other momentary impairments (47). These variables should be taken into account in future studies.

\section{CONCLUSION}

In summary, our results suggest that a significant proportion of patients with schizophrenia can accurately estimate their cognitive skills. Misestimation of cognitive functioning might have been overestimated in individuals with schizophrenia, partly due to psychoaffective confounding factors. The findings of this study confirm the heterogeneous and complex nature of self-awareness and metacognitive abilities in this mental disorder. Caution is warranted before jumping to the conclusion that all individuals with schizophrenia misjudge their cognitive functioning.

\section{REFERENCES}

1. Flavell JH. Metacognitive and cognitive monitoring: A new era of psychological inquiry. Am Psychol (1979) 34:906-1111. doi: 10.1037/0003066X.34.10.906

2. Moritz S, Lysaker PH. Metacognition - What did James H. Flavell really say and the implications for the conceptualization and design of metacognitive interventions. Schizophr Res (2018) 201:20-6. doi: 10.1016/j.schres.2018.06.001

3. Lysaker PH, Dimaggio G. Metacognitive capacities for reflection in schizophrenia: implications for developing treatments. Schizophr Bull (2014) 40(3):487-91. doi: 10.1093/schbul/sbu038

4. Lysaker PH, Minor KS, Lysaker JT, Hasson-Ohayon I, Bonfils K, Hochheiser J, et al. Metacognitive function and fragmentation in schizophrenia: Relationship to cognition, self-experience and developing treatments. Schizophr Res Cogn (2019) 24;19:100142. doi: 10.1016/j.scog.2019.100142

5. Moritz S, Lysaker PH, Hofmann SG, Hautzinger M. Going meta on metacognitive interventions. Expert Rev Neurother (2018) 18(10):739-41. doi: 10.1080/14737175.2018.1520636

6. Beck AT, Baruch E, Balter JM, Steer RA, Warman DM. A new instrument for measuring insight: the Beck Cognitive Insight Scale. Schizophr Res (2004) 68 (2-3):319-29. doi: 10.1016/S0920-9964(03)00189-0

7. Raffard S, D'Argembeau A, Lardi C, Bayard S, Boulenger JP, Van der Linden M. Narrative identity in schizophrenia. Conscious Cogn (2010) 19(1):328-40. doi: 10.1016/j.concog.2009.10.005

8. Amador XF, Strauss DH, Yale SA, Flaum MM, Endicott J, Gorman JM. Assessment of insight in psychosis. Am J Psychiatry (1993) 150(6):873-9. doi: 10.1176/ajp.150.6.873

9. Amador XF, Flaum M, Andreasen NC, Strauss DH, Yale SA, Clark SC, et al. Awareness of illness in schizophrenia and schizoaffective and mood disorders. Arch Gen Psychiatry (1994) 51(10):826-36. doi: 10.1001/archpsyc.1994.03950100074007

10. Lien YJ, Chang HA, Kao YC, Tzeng NS, Yeh CB, Loh CH. Self-Stigma Mediates the Impact of Insight on Current Suicide Ideation in Suicide Attempters with Schizophrenia: Results of a Moderated Mediation Approach. Suicide Life Threat Behav (2018) 48(6):661-76. doi: 10.1111/ sltb. 12384

11. Belvederi Murri M, Amore M, Calcagno P, Respino M, Marozzi V, Masotti M, et al. The "Insight Paradox" in Schizophrenia: Magnitude, Moderators and

\section{DATA AVAILABILITY STATEMENT}

The datasets generated for this study are available on request to the corresponding author.

\section{ETHICS STATEMENT}

The studies involving human participants were reviewed and approved by Comité de Protection des Personnes (CPP) Ile de France IX on January 18, 2010. The patients/participants provided their written informed consent to participate in this study.

\section{AUTHOR CONTRIBUTIONS}

SR conceived and designed the experiments. SR and DC conducted the experiments and collected data. CL, SB analyzed the results. SR and CL wrote the main manuscript text. All authors contributed to the article and approved the submitted version.

Mediators of the Association Between Insight and Depression. Schizophr Bull (2016) 42(5):1225-33. doi: 10.1093/schbul/sbw040

12. Sheffield JM, Karcher NR, Barch DM. Cognitive Deficits in Psychotic Disorders: A Lifespan Perspective. Neuropsychol Rev (2018) 28(4):509-33. doi: 10.1007/s11065-018-9388-2

13. Szöke A, Trandafir A, Dupont ME, Méary A, Schürhoff F, Leboyer M. Longitudinal studies of cognition in schizophrenia: meta-analysis. $\mathrm{Br} \mathrm{J}$ Psychiatry (2008) 192(4):248-57. doi: 10.1192/bjp.bp.106.029009

14. Broadbent DE, Cooper PF, FitzGerald P, Parkes KR. The Cognitive Failures Questionnaire (CFQ) and its correlates. Br J Clin Psychol (1982) 21:1-16. doi: 10.1111/j.2044-8260.1982.tb01421.x

15. Medalia A, Thysen J, Freilich B. Do people with schizophrenia who have objective cognitive impairment identify cognitive deficits on a self report measure? Schizophr Res (2008) 105(1-3):156-64. doi: 10.1016/ j.schres.2008.07.007

16. Stip E, Caron J, Renaud S, Pampoulova T, Lecomte Y. Exploring cognitive complaints in schizophrenia: the subjective scale to investigate cognition in schizophrenia. Compr Psychiatry (2003) 44(4):331-40. doi: 10.1016/S0010440X(03)00086-5

17. Homayoun S, Nadeau-Marcotte F, Luck D, Stip E. Subjective and Objective Cognitive Dysfunction in Schizophrenia - is there a Link? Front Psychol (2011) 2:148. doi: 10.3389/fpsyg.2011.00148

18. Potvin S, Pelletier J, Stip E. Neurocognitive insight in schizophrenia: a metaanalysis. Sante Ment Que (2014) 39(2):183-200. doi: 10.7202/1027839ar

19. Burton CZ, Twamley EW. Neurocognitive insight, treatment utilization, and cognitive training outcomes in schizophrenia. Schizophr Res (2015) 161(23):399-402. doi: 10.1016/j.schres.2014.12.002

20. Burton CZ, Harvey PD, Patterson TL, Twamley EW. Neurocognitive insight and objective cognitive functioning in schizophrenia. Schizophr Res (2016) 171(1-3):131-6. doi: 10.1016/j.schres.2016.01.021

21. Cella M, Swan S, Medin E, Reeder C, Wykes T. Metacognitive awareness of cognitive problems in schizophrenia: exploring the role of symptoms and selfesteem. Psychol Med (2014) 44(3):469-76. doi: 10.1017/S0033291713001189

22. Gould F, McGuire LS, Durand D, Sabbag S, Larrauri C, Patterson TL, et al. Self-assessment in schizophrenia: Accuracy of evaluation of cognition and everyday functioning. Neuropsychology (2015) 29(5):675-82. doi: 10.1037/ neu0000175 
23. Burmester B, Leathem J, Merrick P. Subjective Cognitive Complaints and Objective Cognitive Function in Aging: A Systematic Review and MetaAnalysis of Recent Cross-Sectional Findings. Neuropsychol Rev (2016) 26 (4):376-93. doi: 10.1007/s11065-016-9332-2

24. Gehring K, Taphoorn MJ, Sitskoorn MM, Aaronson NK. Predictors of subjective versus objective cognitive functioning in patients with stable grades II and III glioma. Neurooncol Pract (2015) 2(1):20-31. doi: 10.1093/ nop/npu035

25. Rothlind J, Dukarm P, Kraybill M. Assessment of Self-Awareness of Cognitive Function: Correlations of Self-Ratings with Actual Performance Ranks for Tests of Processing Speed, Memory and Executive Function in Non-Clinical Samples. Arch Clin Neuropsychol (2017) 32(3):316-27. doi: 10.1093/arclin/ acw109

26. Bayard S, Capdevielle D, Boulenger JP, Raffard S. Dissociating self-reported cognitive complaint from clinical insight in schizophrenia. Eur Psychiatry (2009) 24(4):251-8. doi: 10.1016/j.eurpsy.2008.12.010

27. French LM, Lange RT, Brickell T. Subjective cognitive complaints and neuropsychological test performance following military-related traumatic brain injury. J Rehabil Res Dev (2014) 51(6):933-50. doi: 10.1682/ JRRD.2013.10.0226

28. Kay SR, Fiszbein A, Opler LA. The positive and negative syndrome scale (PANSS) for schizophrenia. Schizophr Bull (1987) 13:261-76. doi: 10.1093/ schbul/13.2.261

29. Beck AT, Steer RA, Brown GK. Inventaire de Dépression de Beck - 2ème édition (BDI- II) (Version 2). Paris: Editions du Centre de Psychologie Appliquée (ECPA) (1998).

30. Spielberger CD, Gorsuch RL, Lushene RE. Manual for the State-Trait Anxiety Inventory. Palo Alto, CA: Consulting Psychologists Press (1970).

31. Zimmermann P, Fimm B. Testbatterie zur Aufmerksamkeitsprufung (TAP), Version 2.3. Handbuch. Wurselen: Psytest (2010).

32. Grober E, Buschke H, Crystal H, Bang S, Dresner R. Screening for dementia by memory testing. Neurology (1988) 38:900-3. doi: 10.1212/WNL.38.6.900

33. Cohen J. Statistical Power Analysis for the Behavioral Sciences. New York, NY: Routledge Academic (1988).

34. Moritz S, Ferahli S, Naber D. Memory and attention performance in psychiatric patients: lack of correspondence between clinician-rated and patient-rated functioning with neuropsychological test results. J Int Neuropsychol Soc (2004) 10(4):623-33. doi: 10.1017/S135561770 4104153

35. Donohoe G, Hayden J, McGlade N, O’Gráda C, Burke T, Barry S, et al. Is "clinical" insight the same as "cognitive" insight in schizophrenia? J Int Neuropsychol Soc (2009) 15(3):471-5. doi: 10.1017/S135561770 9090559

36. Potvin S, Aubin G, Stip E. Subjective cognition in schizophrenia. Encephale (2017) F43(1):15-20. doi: 10.1016/j.encep.2016.01.002
37. Addington D, Addington J, Maticka-Tyndale E, Joyce J. Reliability and validity of a depression rating scale for schizophrenics. Schizophr Res (1992) 6(3):201-8. doi: 10.1016/0920-9964(92)90003-N

38. Roth RS, Geisser ME, Theisen-Goodvich M, Dixon PJ. Cognitive complaints are associated with depression, fatigue, female sex, and pain catastrophizing in patients with chronic pain. Arch Phys Med Rehabil (2005) 86(6):1147-54. doi: 10.1016/j.apmr.2004.10.041

39. Hill NL, Mogle J, Wion R, Munoz E, DePasquale N, Yevchak AM, et al. Subjective Cognitive Impairment and Affective Symptoms: A Systematic Review. Gerontologist (2016) 56(6):e109-27. doi: 10.1093/geront/gnw091

40. Malivoire BL, Hare CJ, Hart TL. Psychological symptoms and perceived cognitive impairment in multiple sclerosis: The role of rumination. Rehabil Psychol (2018) 63(2):286-94. doi: 10.1037/rep0000180

41. Yates JA, Clare L, Woods RT, MRC CFAS. Subjective memory complaints, mood and MCI: a follow-up study. Aging Ment Health (2017) 21(3):313-21. doi: 10.1080/13607863.2015.1081150

42. Saperstein AM, Kurtz MM. Current trends in the empirical study of cognitive remediation for schizophrenia. Can J Psychiatry (2013) 58(6):311-8. doi: 10.1177/070674371305800602

43. Medalia A, Lim RW. Self-awareness of cognitive functioning in schizophrenia. Schizophr Res (2004) 71(2-3):331-8. doi: 10.1016/j.schres.2004.03.003

44. Joyce EM, Roiser JP. Cognitive heterogeneity in schizophrenia. Curr Opin Psychiatry (2007) 20(3):268-72. doi: 10.1097/YCO.0b013e3280ba4975

45. Van Rheenen TE, Lewandowski KE, Tan EJ, Ospina LH, Ongur D, Neill E, et al. Characterizing cognitive heterogeneity on the schizophrenia-bipolar disorder spectrum. Psychol Med (2017) 47(10):1848-64. doi: 10.1017/ S0033291717000307

46. Smith EL, Garety PA, Harding H, Hardy A. Are there reliable and valid measures of anxiety for people with psychosis? A systematic review of psychometric properties. Psychol Psychother (2019). doi: 10.1111/papt.12265

47. Moritz S, Klein JP, Desler T, Lill H, Gallinat J, Schneider BC. Neurocognitive deficits in schizophrenia. Are we making mountains out of molehills? Psychol Med (2017) 47(15):2602-12. doi: 10.1017/S00332917

Conflict of Interest: The authors declare that the research was conducted in the absence of any commercial or financial relationships that could be construed as a potential conflict of interest.

Copyright (c) 2020 Raffard, Lebrun, Bayard, Macgregor and Capdevielle. This is an open-access article distributed under the terms of the Creative Commons Attribution License (CC BY). The use, distribution or reproduction in other forums is permitted, provided the original author(s) and the copyright owner(s) are credited and that the original publication in this journal is cited, in accordance with accepted academic practice. No use, distribution or reproduction is permitted which does not comply with these terms. 